\title{
Clinical neurorestorative progress in traumatic brain injury
}

This article was published in the following Dove Press journal:

Journal of Neurorestoratology

20 March 2015

Number of times this article has been viewed

\author{
Huiling Huang' \\ Lin Chen ${ }^{2,3}$ \\ Hongyun Huang ${ }^{4-6}$ \\ 'Tianjin Key Laboratory of Cerebral \\ Vascular and Neurodegenerative \\ Diseases, Tianjin Huanhu Hospital, \\ Tianjin Neurosurgical Institute, Tianjin, \\ People's Republic of China; ${ }^{2}$ Medical \\ Center, Tsinghua University, Beijing, \\ People's Republic of China; ${ }^{3} T$ singhua \\ University Yuquan Hospital, Beijing, \\ People's Republic of China; ${ }^{4}$ General \\ Hospital of Chinese people's Armed \\ Police Forces, ${ }^{5}$ Beijing Rehabilitation \\ Hospital of Capital Medical University, \\ Beijing, People's Republic of China; \\ ${ }^{6}$ Beijing Hongtianji Neuroscience \\ Academy, Beijing, People's Republic \\ of China
}

Correspondence: Huiling Huang No 122 Qixiangtai Road, Hexi District, Tianjin, 300060, People's Republic of China Email huanghuiling@I26.com

Lin Chen

No 5 Shijingshan Road, Shijingshan

District, Beijing 100040, People's Republic of China

Email chenlin_china@I63.com

\begin{abstract}
Traumatic brain injury (TBI) is a leading cause of death and disability from trauma to the central nervous system. Besides the surgical interventions and symptomatic management, the conventional therapies for TBI and its sequelae are still limited. Recently emerging evidence suggests that some neurorestorative treatments appear to have a potential therapeutic role for TBI and improving the patient's quality of life. The current clinical neurorestorative strategies available in TBI include pharmacological treatments (recombinant human interleukin-1 receptor antagonist, amantadine, lithium, and valproate), the neuromodulation treatments (repetitive transcranial magnetic stimulation, transcranial direct current stimulation, and low-level laser therapy), cell transplantation (bone marrow stromal cells and umbilical cord stromal cells), and combined neurorehabilitation. In this review, we summarize the recent clinical neurorestorative progress in the management of neurodegeneration as well as cognitive and motor deficits after TBI; indeed further clinical trials are required to provide more robust evidence.
\end{abstract}

Keywords: brain trauma, neurorestorative treatment, cell transplantation, clinical study

\section{Introduction}

Nowadays traumatic brain injury (TBI) remains one major source of morbidity and mortality with impairment in sensory, motor, language, emotional processing, and cognitive functions. The principal mechanisms of TBI are classified as 1) focal brain damage due to contact injury types resulting in contusion, laceration, and intracranial hemorrhage or 2) diffuse brain damage due to acceleration/deceleration injury types leading to diffuse axonal injury or brain swelling.

Outcome of head injury is determined by two substantially different mechanisms/ stages: the primary insult and the secondary insult. ${ }^{1}$ The multidimensional cascade of secondary brain injury commonly develops. It is influenced by changes in cerebral blood flow (hypo- and hyperperfusion), impairment of cerebrovascular autoregulation, cerebral metabolic dysfunction and inadequate cerebral oxygenation. The main secondary pathological mechanisms encompass excitotoxicity, ionic imbalances, inflammatory response, oxidative stress, and apoptosis. ${ }^{2}$ Diffusion tensor imaging studies also reveal the decreased cellular integrity, particularly in the corpus callosum. ${ }^{3}$

The injured brain response can activate endogenous reparative processes for countering the neurodegeneration or remodeling of the brain in order to enhance functional recovery, ${ }^{4}$ which includes angiogenesis, neurogenesis, synaptogenesis, oligodendrogenesis, and axonal remodeling. However, the endogenous neurorestoration following TBI is limited. ${ }^{5}$ Neurorestorative strategies in preclinical studies provide promising opportunities for 
clinical TBI treatment. ${ }^{6}$ Here we briefly summarize the clinical neurorestorative progress in TBI.

\section{Clinical neurorestorative progress Pharmacological therapies}

Despite strong preclinical evidence supporting pharmacological neuroprotection treatment to reduce secondary injury, more than 30 clinical trials of neuroprotective drugs in TBI patients to date have failed (http://www.clinicaltrials.gov). It should be emphasized that TBI is a highly complex disorder and may require markedly different treatments. So a reappraisal of clinical trial methodology in TBI is required. ${ }^{7}$ The future pharmacological management of TBI patients needs to combine neuroprotective drugs with compounds enhancing regeneration.

\section{Recombinant human interleukin- I receptor antagonist (IL-I ra)}

First a single center, Phase II, open label, randomized controlled study in severe TBI, with IL-1ra at a dose of $100 \mathrm{mg}$ subcutaneously once a day for 5 days in 20 patients, showed safety, brain penetration, and a modification of the neuro-inflammatory response to TBI by putative neuroprotection. ${ }^{8}$

\section{Amantadine}

A patient with diffuse axonal injury-associated TBI was started on amantadine (AMD) in the first 3 months after injury showing a consistent trend toward a more rapid functional improvement. ${ }^{9}$ A multicenter, double-blind, randomized, placebo-controlled trial of AMD, at a dose of 100-200 mg for 4 weeks, resulted in acceleration of the pace of recovery during acute rehabilitation in 184 patients with prolonged post-traumatic disturbances in consciousness. ${ }^{10}$

\section{Lithium}

In fact, lithium has long been used in a clinical setting to treat manic depression, granulocytopenia resulting from radiation and chemotherapy, to boost immunoglobulins after vaccination, and to enhance natural killer activity. Recent studies revealed that lithium may ameliorate physiological as well as cognitive deficits induced by TBI in clinical research. ${ }^{11}$

\section{Valproate}

Recent studies suggest that some anticonvulsants (ie, valproate [VPA], carbamazepine, and lamotrigine) may be useful in TBI patients. VPA also exhibits improved recovery of brain function and prevention of late-onset epilepsy after severe TBI. ${ }^{12,13}$

\section{Neuromodulation and rehabilitation}

Noninvasive brain stimulation includes repetitive transcranial magnetic stimulation, transcranial direct current stimulation, and low-level laser therapy, which may improve the function of sequelae after brain injury and have been proven to be safe and feasible for treatment of TBI. ${ }^{14}$ Enriched environment and voluntary physical exercise show promise in promoting functional outcome after TBI. In the chronic stage, brain stimulation coupled with rehabilitation may enhance behavioral recovery, learning of new skills, guide processes of cortical reorganization, and enable functional restoration in TBI. ${ }^{15}$

Early rehabilitation has been shown to improve functional outcomes; the rehabilitation program itself has to be based on real-world demands and experiences. Rehabilitation has to be continued beyond the post-acute stage in order to promote neuronal reorganization, monitor the children's development as well as identify and manage new issues that may appear with growth, development, and maturation. ${ }^{16}$ Enriched environment, including prolonged and intensive physical activity - possibly combined with juggling training and intensive cognitive stimulation will improve recovery from TBI.

Peters reported that 20 days ( 5 days a week for 4 weeks), with 150 minutes intervention per day of repetitive, taskspecific training equally divided among balance; gait training; strength; coordination; and range of motion, resulted in improvements in walking speed, mobility, and balance post-intervention and maintained gains in fast walking speed and mobility at 3 months. ${ }^{17}$

\section{Cell therapy}

Treatment of damaged brain with bone marrow stromal cells (BMSCs) promotes functional recovery and facilitates central nervous system endogenous plasticity and remodeling. ${ }^{18}$ The potential of cell biodelivery as a novel cell-therapeutic approach has been explored in patients. ${ }^{19}$ It was reported that the clinical outcome of autologous BMSC therapy for TBI via lumbar puncture. ${ }^{20}$ Ninety-seven patients ( 24 in persistent vegetative state and 73 with disturbed motor activity) were treated. Two weeks later, 38 of 97 patients $(39.2 \%)$ improved their functions. Eleven of 24 patients $(45.8 \%)$ in persistent vegetative state had improved consciousness. Twenty-seven of 73 patients $(37.0 \%)$ with a motor disorder began to improve their motor functions. It demonstrated the safety and potential central nervous system structural preservation effect of intravenous autologous bone marrow mononuclear cells after severe TBI in children (aged 5 to 14 years) in the absence of controlled trials. ${ }^{21}$ Young patients improved more easily than elders. The earlier the cellular transplant begins 
in the subacute stage of TBI, the better the results. Seven TBI patients were treated by autologous BMSC transplantation. ${ }^{22}$ A primary administration of $10^{7}-10^{9}$ cells was applied directly to the injured area during the cranial operation; a second dose of $10^{8}-10^{10}$ cells was infused intravenously. Neurologic function (evaluation with Barthel Index) was significantly improved during the following 6 months.

Twenty patients with sequelae of TBI were treated with umbilical cord mesenchymal stem cells transplantation through lumbar puncture $\left(1 * 10^{7}\right.$ stem cells, four times in 5-7 days). ${ }^{23}$ Fugl-Meyer Assessments and Functional Independence Measures before and at 6 months post-transplantation in TBI patients showed improvement in the neurological function (the sub-score of motor, sensation, balance), and significant improvement in sub-score of self-care, sphincter control, mobility, locomotion, communication, and social cognition compared to the control group. A combination therapy of human umbilical cord blood cells and granulocyte-colony stimulating factor synergistically diminished TBI-induced neuro-inflammation and stimulated endogenous neurogenesis in TBI. ${ }^{24}$ Granulocyte-colony stimulating factor might have produced a conducive micro-environment for the transplanted human umbilical cord blood cells to integrate with the host tissue.

\section{Discussion}

\section{The possible mechanisms of neurorestorative drugs \\ IL-I ra}

The cytokine IL (IL-1) mediates ischemic brain damage. The endogenous IL-1 receptor competitive antagonist (IL-1ra) protects against ischemic injury. IL-1ra causes a marked reduction of cell death when administered peripherally or at a delay in transient cerebral ischemia in the rat and appeared to be safe and well tolerated in acute stroke patients. ${ }^{25} \mathrm{IL}-1 \mathrm{ra}$ confers neuroprotection of neuronal injury in several rodent models including trauma, stroke, and excitotoxicity. ${ }^{8}$

\section{AMD}

AMD is an antagonist of the N-methyl-D-aspartate-type glutamate receptor, increases dopamine release, blocks dopamine reuptake, and has an inhibitory effect on microglial activation and neuro-inflammation. It can ameliorate dopamine-release deficits as well as cognitive and motor deficits caused by cerebral fluid-percussion injury in the rats. ${ }^{26}$ The clinically therapeutic doses $(100-400 \mathrm{mg} /$ day) of AMD afford neuroprotection and significantly beneficial effects on cognitive outcome and neurobehavioral sequelae after experimental TBI. ${ }^{27}$ The mechanisms of AMD effects are still poorly understood making animal studies of its effects an important step in improving clinical use.

\section{Lithium}

Lithium is a primary medicine for bipolar disorder, has been known to have neuroprotective effects, and stimulates neurogenesis in TBI via multiple signaling pathways. It upregulates neurotrophins and growth factors (eg, brainderived neurotrophic factor $[\mathrm{BDNF}])$, modulates inflammatory molecules, upregulates neuroprotective factors, and downregulates pro-apoptotic factors. ${ }^{11}$ Literature shows that TBI-induced neuronal death, microglial activation, and cyclooxygenase- 2 induction can be attenuated by lithium. Lithium reduces TBI-induced matrix metalloproteinase-9 expression and preserved the integrity of the blood-brain barrier. In addition, lithium's protective effects are triggered by increasing phosphorylation of glycogen synthase kinase-3 $\beta$ (GSK-3 $\beta)$ and thereby inhibiting its activity. ${ }^{28}$

\section{Valproic acid}

Valproic acid is a widely used antiepileptic drug, whose novel neuroprotective effects have been shown recently in the animal models of TBI. Valproic acid protects the brain from injury progression via anti-inflammatory, anti-apoptotic, and neurotrophic effects, which act on the many targets including gamma-aminobutyric acid (GABA) transaminase, voltagegated sodium channels, GSK-3, and histone deacetylases..$^{29,30}$

\section{The possible neurorestorative mechanisms of neuromodulation treatments and rehabilitation}

The mechanisms of neuromodulation treatments and rehabilitation include: 1) decrease the cortical hyperexcitability of acute TBI; 2) modulate long-term synaptic plasticity as to avoid maladaptive consequences; and 3) combined with physical and behavioral therapy, facilitate cortical reorganization and consolidation of learning in specific neural networks. ${ }^{31}$

\section{The possible neurorestorative mechanisms of BMSCs}

Cell transplantation can rebuild damaged brain cell circuits and restore lost neurological functions in a brain injury. Findings of in vitro and in vivo studies show that BMSCs have an ability to secrete an array of growth factors and cytokines and probably differentiate into cells of multiple tissues, including neurons and glial cells, which have an influence on repair of damaged tissue. ${ }^{32}$ 
A significant amount of preclinical research has evaluated the therapeutic use of BMSCs to treat TBI. ${ }^{18}$ The cells could survive, migrate to the area of injury, express neural cell markers and even improve motor function up to 4 weeks post-transplantation A series of research papers reported on BMSCs treatment of rodent TBI with various cell donors (rats, mice or humans), with different cellular doses, various delivery routes (intravenous, intra-arterial, intracerebral, intrathecal, and intracisternal), with or without neurotrophic factors, the acute and delayed therapeutic window (ie, 1 day, 1 week, and 1 month) and rodent ages. ${ }^{33-35}$ BMSCs administered intravenously could significantly reduce the neurological severity scores in experimental animals. ${ }^{36}$ Intracerebral administration of BMSCs after TBI could promote endogenous cellular proliferation and increase the expression of growth factors such as NGF or BDNF. ${ }^{37}$ A number of bromodeoxyuridine-positive BMSCs increased neurogenesis possibly in the sub-ventricular zone, hippocampus and pericontusional area after 2 weeks post-injury. ${ }^{38}$ These data suggest that transplanted BMSCs work as "small molecular factories" by secreting neurotrophins, growth factors, and other supportive substances (such as IGF1, VEGF, NGF, BDNF, EGF, and NT3-4) after brain injury, which may continually produce therapeutic benefits in the damaged brain and behavioral/histological improvement. ${ }^{39}$

One week after TBI, BMSC therapy was conducted which led to long-term functional recovery and the cells remained in the brain for 3 months after treatment. By delayed intracerebral transplantation of BMSC, 2 months after controlled cortical impact (CCI), BMSCs still survived in the host tissue and some of them showed expression of Neu-N or GFAP. This result suggested that neuronal and astroglial transdifferentiation, and endogenous neurogenesis took place. ${ }^{40}$

Human BMSCs administered intravenously 24 hours after TBI were also shown to migrate to the injured brain, express neural phenotypes, survive for 3 months, and improve functional outcomes that may be attributed to stimulation of endogenous neurorestorative functions such as neurogenesis and synaptogenesis. ${ }^{41}$ By using magnetic resonance imaging (MRI) on human BMSCs labeled with superparamagnetic iron oxide (SPIO) nanoparticles in vivo, it could be observed that treatment with hBMSCs following TBI could diminish hemodynamic abnormalities, reduce generalized cerebral atrophy, and improve functional outcomes by early restoration and preservation of cerebral blood flow in the brain regions adjacent to and remote from the impact site. ${ }^{42}$ BMSCs transfected with adenovirus green fluorescent protein (Ad-GFP) after transplanted intrathecally into an autologous rabbit model of TBI could also migrate to the TBI site and improve motor dysfunction..$^{33}$ One of the mechanisms of action of these cellular therapies appears to rely upon the ability of the cells to influence microglia/macrophage phenotype and alter the state of the inflammatory response. ${ }^{43}$ Contrary to the positive coverage, however, ${ }^{44}$ systemic human MSC transplantation administered intravenously 24 hours after middle cerebral artery occlusion (MACO) proved to have a weak neurogenic and neuroprotective effect because human MSC were detected robustly in lung, spleen, kidney, and intestine, but not in the brain.

\section{The possible neurorestorative mechanisms of umbilical cord blood cells or stromal cells}

The infusion of human umbilical cord blood cells into the tail veins of rats 24 hours after CCI, showed that the cells migrated to the parenchyma of the injured brain, expressed the neuronal markers (NeuN and MAP-2) and the astrocytic marker (GFAP), and significantly reduced motor and neurological deficits. ${ }^{45}$ Evidence has demonstrated that umbilical cord stromal or placental tissue, rather than cord blood, can offer a potentially non-invasive and readily available source of large amounts of human stromal cells. ${ }^{46,47}$ This heterogeneous mononuclear fraction, when stimulated with basic fibroblast growth factor (bFGF) and human epidermal growth factor (hEGF), has been shown to differentiate into cells that are positive for the neural markers beta-tubulin III (TUJ1) and glial fibrillary acidic protein (GFAP) ${ }^{48}$ It could stimulate the injured brain and evoke trophic events, microglia/macrophage phenotypical switch, and glial scar inhibitory affects that remodel the brain and lead to significant improvement of neurologic outcome after CCI in mice models. ${ }^{49}$

In summary, cell-based neurorestorative strategies including pharmacological agents, neuromodulation, and rehabilitation, have a pivotal role in recovering some lost functions. The transplanted cells appear to have a knack for integrating into damaged circuits and transforming into needed cell types to protect the brain from further damage of TBI. Even cell therapy for TBI is in the early phase, the future therapeutic regimes also should be aimed at suppressing aberrant integration that may contribute to co-morbidities such as epilepsy or cognitive impairment. ${ }^{50}$

\section{Acknowledgment}

We thank Ms Lu Zheng and Jiangfeng Guo for preparing this manuscript. 


\section{Disclosure}

The authors have no conflicts of interest to disclose.

\section{References}

1. Werner C, Engelhard K. Pathophysiology of traumatic brain injury. Br J Anaesth. 2007;99(1):4-9.

2. Freire MA. Pathophysiology of neurodegeneration following traumatic brain injury. West Indian Med J. 2012;61(7):751-755.

3. Keightley ML, Sinopoli KJ, Davis KD, et al. Is there evidence for neurodegenerative change following traumatic brain injury in children and youth? A scoping review. Front Hum Neurosci. 2014;8:139.

4. Schoch KM, Madathil SK, Saatman KE. Genetic manipulation of cell death and neuroplasticity pathways in traumatic brain injury. Neurotherapeutics. 2012;9(2):323-337.

5. Xiong Y, Mahmood A, Meng Y, et al. Neuroprotective and neurorestorative effects of thymosin $\beta 4$ treatment following experimental traumatic brain injury. Ann N Y Acad Sci. 2012;1270:51-58.

6. Xiong Y, Mahmood A, Chopp M. Neurorestorative treatments for traumatic brain injury. Discov Med. 2010;10(54):434-442.

7. Miller G. Neuroscience. New guidelines aim to improve studies of traumatic brain injury. Science. 2010;328(5976):297.

8. Helmy A, Guilfoyle MR, Carpenter KL, et al. Recombinant human interleukin-1 receptor antagonist in severe traumatic brain injury: a phase II randomized control trial. J Cereb Blood Flow Metab. 2014;34(5):845-851

9. Meythaler JM, Brunner RC, Johnson A, Novack TA. Amantadine to improve neurorecovery in traumatic brain injury-associated diffuse axonal injury: a pilot double-blind randomized trial. $J$ Head Trauma Rehabil. 2002;17(4):300-313.

10. Giacino JT, Whyte J, Bagiella E, et al. Placebo-controlled trial of amantadine for severe traumatic brain injury. $N$ Engl J Med. 2012;366(9) 819-826.

11. Leeds PR, Yu F, Wang Z, et al. A new avenue for lithium: intervention in traumatic brain injury. ACS Chem Neurosci. Epub April 11, 2014.

12. TaiYT, Lee WY, Lee FP, et al. Low dose of valproate improves motor function after traumatic brain injury. Biomed Res Int. 2014;2014:980657.

13. Ma CY, Xue YJ, Li M, Zhang Y, Li GZ. Sodium valproate for prevention of early posttraumatic seizures. Chin J Traumatol. 2010;13(5): 293-296.

14. Pape TL, Rosenow JM, Patil V, et al. rTMS Safety for Two Subjects With Disordered Consciousness After Traumatic Brain Injury. Brain Stimul. 2014;7(4):620-622.

15. Demirtas-Tatlidede A, Vahabzadeh-Hagh AM, Bernabeu M, Tormos JM, Pascual-Leone A. Noninvasive brain stimulation in traumatic brain injury. J Head Trauma Rehabil. 2012;27(4):274-292.

16. Yen HL, Wong JT. Rehabilitation for traumatic brain injury in children and adolescents. Ann Acad Med Singapore. 2007;36(1):62-66.

17. Peters DM, Jain S, Liuzzo DM, et al. Individuals with chronic traumatic brain injury improve walking speed and mobility with intensive mobility training. Arch Phys Med Rehabil. 2014;95(8):1454-1460.

18. Li Y, Chopp M. Marrow stromal cell transplantation in stroke and traumatic brain injury. Neurosci Lett. 2009;456(3):120-123.

19. Heile A, Brinker T. Clinical translation of stem cell therapy in traumatic brain injury: the potential of encapsulated mesenchymal cell biodelivery of glucagon-like peptide-1. Dialogues Clin Neurosci. 2011;13(3):279-286.

20. Tian C, Wang X, Wang X, et al. Autologous bone marrow mesenchymal stem cell therapy in the subacute stage of traumatic brain injury by lumbar puncture. Exp Clin Transplant. 2013;11(2):176-181.

21. Cox CS Jr, Baumgartner JE, Harting MT, et al. Autologous bone marrow mononuclear cell therapy for severe traumatic brain injury in children. Neurosurgery. 2011;68(3):588-600.

22. Zhang ZX, Guan LX, Zhang K, Zhang Q, Dai LJ. A combined procedure to deliver autologous mesenchymal stromal cells to patients with traumatic brain injury. Cytotherapy. 2008;10(2):134-139.
23. Wang S, Cheng H, Dai G, et al. Umbilical cord mesenchymal stem cell transplantation significantly improves neurological function in patients with sequelae of traumatic brain injury. Brain Res. 2013;1532:76-84.

24. Dela Peña I, Sanberg P, Acosta S, et al. Stem cells and G-CSF for treating neuroinflammation in traumatic brain injury: aging as a co-morbidity factor. J Neurosurg Sci. 2014;58(3):145-149.

25. Emsley HC, Smith CJ, Georgiou RF, et al. A randomised phase II study of interleukin-1 receptor antagonist in acute stroke patients. J Neurol Neurosurg Psychiatry. 2005;76(10):1366-1372.

26. Huang EY, Tsui PF, Kuo TT, et al. Amantadine ameliorates dopaminereleasing deficits and behavioral deficits in rats after fluid percussion injury. PLoS One. 2014;9(1):e86354.

27. Wang T, Huang XJ, Van KC, et al. Amantadine improves cognitive outcome and increases neuronal survival after fluid percussion traumatic brain injury in rats. J Neurotrauma. 2014;31(4):370-377.

28. Yu F, Wang Z, Tchantchou F, et al. Lithium ameliorates neurodegeneration, suppresses neuroinflammation, and improves behavioral performance in a mouse model of traumatic brain injury. $J$ Neurotrauma. 2012;29(2):362-374.

29. Chen S, Wu H, Klebe D, Hong Y, Zhang J. Valproic Acid: a new candidate of therapeutic application for the acute central nervous system injuries. Neurochem Res. 2014;39(9):1621-1633.

30. Dash PK, Orsi SA, Zhang M, et al. Valproate administered after traumatic brain injury provides neuroprotection and improves cognitive function in rats. PLoS One. 2010;5(6):e11383.

31. Villamar MF, Santos Portilla A, Fregni F, Zafonte R. Noninvasive brain stimulation to modulate neuroplasticity in traumatic brain injury. Neuromodulation. 2012;15(4):326-338.

32. Opydo-Chanek M. Bone marrow stromal cells in traumatic brain injury (TBI) therapy: true perspective or false hope? Acta Neurobiol Exp (Wars). 2007;67(2):187-195.

33. Liu W, Jiang X, Fu X, et al. Bone marrow stromal cells can be delivered to the site of traumatic brain injury via intrathecal transplantation in rabbits. Neurosci Lett. 2008;434(2):160-164.

34. Mahmood A, Lu D, Qu C, Goussev A, Chopp M. Long-term recovery after bone marrow stromal cell treatment of traumatic brain injury in rats. J Neurosurg. 2006;104(2):272-277.

35. Qu C, Mahmood A, Lu D, et al. Treatment of traumatic brain injury in mice with marrow stromal cells. Brain Res. 2008;1208:234-239.

36. Mahmood A, Lu D, Chopp M. Intravenous administration of marrow stromal cells (MSCs) increases the expression of growth factors in rat brain after traumatic brain injury. J Neurotrauma. 2004;21(1):33-39.

37. Lu D, Li Y, Mahmood A, et al. Neural and marrow-derived stromal cell sphere transplantation in a rat model of traumatic brain injury. J Neurosurg. 2002;97(4):935-940.

38. Mahmood A, Lu D, Chopp M. Marrow stromal cell transplantation after traumatic brain injury promotes cellular proliferation within the brain. Neurosurgery. 2004;55(5):1185-1193.

39. Chen Q, Long Y, Yuan X, et al. Protective effects of bone marrow stromal cell transplantation in injured rodent brain: synthesis of neurotrophic factors. J Neurosci Res. 2005;80(5):611-619.

40. Bonilla C, Zurita M, Otero L, Aguayo C, Vaquero J. Delayed intralesional transplantation of bone marrow stromal cells increases endogenous neurogenesis and promotes functional recovery after severe traumatic brain injury. Brain Inj. 2009;23(9):760-769.

41. Mahmood A, Lu D, Qu C, Goussev A, Chopp M. Human marrow stromal cell treatment provides long-lasting benefit after traumatic brain injury in rats. Neurosurgery. 2005;57(5):1026-1031.

42. Li L, Jiang Q, Qu CS, et al. Transplantation of marrow stromal cells restores cerebral blood flow and reduces cerebral atrophy in rats with traumatic brain injury: In vivo MRI study. J Neurotrauma. 2011;28(4): 535-545.

43. Hetz RA, Bedi SS, Olson S, et al. Progenitor cells: therapeutic targets after traumatic brain injury. Transl Stroke Res. 2012;3(3):318-323.

44. Steiner B, Roch M, Holtkamp N, et al. Systemically administered human bone marrow-derived mesenchymal stem home into peripheral organs but do not induce neuroprotective effects in the MCAo-mouse model for cerebral ischemia. NeurosciLett. 2012;513(1):25-30. 
45. Lu D, Sanberg PR, Mahmood A, et al. Intravenous administration of human umbilical cord blood reduces neurological deficit in the rat after traumatic brain injury. Cell Transplant. 2002;11(3):275-281.

46. Secco M, Zucconi E, Vieira NM, et al. Multipotent stem cells from umbilical cord: cord is richer then blood! Stem Cells. 2008;26(1): $146-150$.

47. Newcomb JD, Willing AE, Sanberg PR. Umbilical cord blood cells. Methods Mol Biol. 2009;549:119-136.
48. Zigova T, Song S, Willing AE, et al. Human umbilical cord blood cells express neural antigens after transplantation into the developing rat brain. Cell Transplant. 2002;11(3):265-274.

49. Zanier ER, Montinaro M, Vigano M, et al. Human umbilical cord blood mesenchymal stem cells protect mice brain after trauma. Crit Care Med. 2011;39(11):2501-2510.

50. Kernie SG, Parent JM. Forebrain neurogenesis after focal Ischemic and traumatic brain injury. Neurobiol Dis. 2010;37(2):267-274.

\section{Publish your work in this journal}

The Journal of Neurorestoratology is an international, peer-reviewed, open access online journal publishing original research and review articles on the subject of Neurorestoratology. To provide complete coverage of this revolutionary field the Journal of Neurorestoratology will report on relevant experimental research, technological advances, and

\section{Dovepress}

clinical achievements. The manuscript management system is completely online and includes a very quick and fair peer-review system, which is all easy to use. Visit http://www.dovepress.com/testimonials.php to read real quotes from published authors.

Submit your manuscript here: http://www.dovepress.com/journal-of-neurorestoratology-journal 